\title{
HEAVY METALS IN RECYCLED PASTRY PACKAGES AND PASTRIES
}

\author{
I. MOHAMMADPOUR ${ }^{a}$, R. AHMADKHANIHA ${ }^{b}$, M. ZARE JEDDI ${ }^{\mathrm{c}}$ and N. RASTKARI ${ }^{* *}$ \\ ${ }^{\mathrm{a}}$ Center for solid waste research (CSWR), Institute for environmental research, Tehran University of Medical \\ Sciences (TUMS), No. 1547, North Kargar Avenue, Enghelab Square, Tehran. Iran \\ ${ }^{b}$ Department of Human Ecology, School of Public Health, Tehran University of Medical Sciences, Ghods St., \\ Poursina St., PO Box 1417613151, Tehran. Iran \\ ${ }^{\mathrm{c}}$ Center for Air Pollution Research (CAPR), Institute for Environmental Research (IER), Tehran University of \\ Medical Sciences, No. 1547, North Kargar Avenue, Enghelab Square, Tehran. Iran
}

(Received: 22 January 2016; accepted 22 April 2016)

The safe use of recycled paper and board material for food packaging applications is a major area of investigations. Therefore, the aim of current study was to evaluate the amount of heavy metals in recycled paper pastry packages and measurement of migration over the time into pastries.

In this study, the presence of heavy metals in common confectionery packaging and effects of storage time and kind of pastry on the migration are investigated.

The results of the study indicate that harmful metals such as $\mathrm{Pb}$ are detected at high concentrations in most of the recycled boxes that are used for pastry packaging, and heavy metals could migrate from the recycled packages into food during usage.

It seems that the procedure of preparing recycled paperboard for food packaging should be reconsidered.

Keywords: recycled paper, packaging materials, heavy metals, food

The food industry uses a huge amount of cellulose fibres in the manufacturing of food packaging. Paper and paper derivatives are indeed one of the primary sources of food packaging all over the world, and their use is promoted not only by economic factors (paper packaging is extremely cheap) but also by their safety of use. Paper and board are light and inexpensive materials, widely used for packaging dry foods (BINDERUP et al., 2002; MANALILI et al., 2011).

To protect the environment, the interest in the use of recycled products has been growing for the recent years. Recycled paper and boards are mainly used in direct contact with dry foodstuffs. The use of recycled paper for the making of food packaging paper raises some queries concerning the possible toxicological potential of these products. However, packaging may have a significant effect on food quality and safety by the transfer of undesirable compounds from packaging into the food, so that the scientific and the political community has been forced to reconsider the safety of using recycled paper for food packaging, and to define standards of quality for secondary cellulose fibres to be used for the production of food packaging. Recycled paper usually has a less predictable composition and more inferior functional properties than boards made with virgin fibres (EC, 2004, 2006). Depending on the production process and the use of paper before it is collected for recycling, it is exposed to numerous compounds that are harmful for the human health. Many of these compounds

\footnotetext{
* To whom correspondence should be addressed.

Phone: +98 21 88978395; fax: +98 21 88978398, e-mail: nr_rastkari@yahoo.com
}

0139-3006/\$20.00 @ 2016 Akadémiai Kiadó, Budapest 
are possible to be found in paper and paperboard after the process of recycling (СНALвот et al., 2006; Suciu et al., 2013).

Regarding food safety, recycled packaging materials need to comply with specific legislative requirements. A recently approved proposal by the Council of Europe requires the control and analysis of a series of contaminants in recycled paper and board such as heavy metals, plasticizers, aromatic amines, poly aromatic hydrocarbons, benzophenone, etc. (NeRín \& Asensio, 2007). Heavy metals are important environmental pollutants, and their toxicity is a problem of increasing significance for ecological, nutritional, and environmental reasons (KHAN et al., 2013). Metal pollution has harmful effect on biological systems and does not undergo biodegradation. So they can be accumulated in living organisms, thus causing various diseases and disorders even in relative lower concentrations (TANGAHU et al., 2011). To our knowledge, there is only one report about determination of heavy metals in dry foodstuff packaging (KIM et al., 2008). However, in that study the authors determined heavy metals in candy packages and candies regardless of the origin of the cardboard used for packaging. The aim of this study was to survey the presence of heavy metals in common confectionary packaging from local market in Tehran, Iran. In addition, the effects of storage time and kind of pastry on the migration of heavy metals were investigated.

\section{Materials and methods}

Thirty recycled pastry boxes (grammage: 300-350 $\mathrm{g} \mathrm{m}^{-2}$ ) the inner layer of which were covered with polyethylene film and were used for direct pastry packaging were purchased from different confectionary stores situated in different parts of the city in winter and spring 2013. In additional sampling, four pastry samples of each group of pastry (Danish pastry, marmalade pastry, puffy pastry, and sugar coated biscuit), totally 16 samples, were purchased from retail outlets in Tehran. Purchasing of the samples was carried out in March 2013. The selected pastry boxes were similar in shape with dimensions $23 \times 23 \times 7 \mathrm{~cm}^{3}$ and capacity of $1 \mathrm{~kg}$. The pastry pieces are usually packaged in the way that all of them contact the sides of the boxes. One third of each sample was pooled, homogenized, and digested on the same day of purchase, and used directly for analyses at time zero. The remaining contents of each sample were stored in the same recycled boxes at room temperature and analysed after different storage times (5 and 10 days). To make a reproducible sampling, in each sampling run, the pastry pieces were selected from different parts of the boxes. All reagents used in present work were of analytical grade. Double deionized water was used for all dilutions. All the glass wares were washed with chromic acid, soaked in 5\% nitric acid and rinsed with distilled water prior to use. The standard solution of investigated metal ions for calibration procedure was prepared by diluting a stock solution of $1.000 \mathrm{mg} \mathrm{l}^{-1}$ of all the investigated elements supplied by Merck. An Optical Emission Spectrometry with inductively coupled plasma excitation (ICP-OES), Company: Spectro, Germany made, model Spectro Arcos with a sequential mode of operation was used in the present study. The separating parameters for the investigated elements were set as recommended by the manufacturer.

Wet digestion of different kinds of pastry was carried out by digesting each sample with a mixture of $\mathrm{HNO}_{3}: \mathrm{H}_{2} \mathrm{SO}_{4}(8: 4)$. This mixture was heated up to $130^{\circ} \mathrm{C}$ for $1 \mathrm{~h}$. After cooling, $5 \mathrm{ml}$ of distilled water was added to the sample and mixed. The residue was filtered. The sample was then diluted to $100 \mathrm{ml}$ with distilled water (JAGGI \& GuPTA, 2013). Blank digestions were also performed in the same way. The levels of analytes in the final solutions 
were determined by ICP-OES. All sample solutions were run in triplicates and were clear. A recovery test of the total analytical procedure was carried out for some of the metals in selected samples by spiking analysed samples with aliquots of metal standards and then reanalysing the samples.

Pastry boxes were digested using the dry ash method $\left(500{ }^{\circ} \mathrm{C}, 6 \mathrm{~h}\right)$. Samples were weighted to approximately $0.2 \mathrm{~g}$ in ceramic crucible and diluted to $10-100 \mathrm{ml}$ with $0.5 \mathrm{~mol} \mathrm{l}^{-1} \mathrm{HNO}_{3}$ after digesting. Sample blanks were also performed with empty ceramic crucible.

All statistical analyses were carried out by SPSS for Windows (version 11.5). The data determined were expressed as the mean of three replicate determinations and presented as means \pm SD. Data from each treatment or control group were analysed for differences by using repeated-measures analysis. Tukey's all-pairwise-comparison test was used to identify differences between different groups. $\mathrm{P}<0.05$ was considered statistically significant.

\section{Results and discussion}

In the present study, 30 commercially available recycled pastry boxes were checked for the presence of 10 heavy metals, which are usually found as residues of the recycling procedures, so as to evaluate their suitability to be used as packaging materials in direct contact with foodstuffs.

Table 1 shows the concentrations of heavy metals found in the analysis of the 30 box samples by ICP-OES. Results were compared with the proposed limits in the industry guideline for the compliance of paper and board materials and articles for food contact by Iranian National Standardization Organization (INSO, 2015). Since in this case the maximum limit is expressed as weight/area unit, whereas the results obtained by an analytical measurement provided the weight/weight results, a conversion to weight/area units had to be done. The conversion was done by taking into account the actual grammage of analysed paper (Equation 1) (JAMNICKI et al., 2012):

$$
Q_{a}=\frac{Q_{m} \times G}{10^{5}}
$$

where:

$Q_{a}$ is concentration of substance in paper expressed as $\mathrm{mg} \mathrm{dm}^{-2}$,

$\mathrm{Q}_{\mathrm{m}}$ is concentration of substance in paper expressed as $\mathrm{mg} \mathrm{kg}^{-1}$,

$\mathrm{G}$ is grammage of paper expressed as $\mathrm{g} \mathrm{m}^{-2}$,

$\mathrm{Q}_{\mathrm{a}}$ is the maximum quantity of the contaminant allowed in the packaging, if it is assumed that $100 \%$ of it will migrate into the foodstuff.

The results of detected heavy metals in analysed box samples and permitted concentrations expressed as $\mathrm{mg} \mathrm{dm}^{-2}$ of material (Table 1). As was detected at extremely low levels in all samples. Moreover, $\mathrm{Hg}$ was detected in none of the samples. Cd was found in all samples, but well below the proposed limits. Pb levels of over $0.003 \mathrm{mg} \mathrm{dm}^{-2}$ was detected in 28 out of 30 samples. $\mathrm{Pb}$ may be present in cardboard food-packaging materials as a residue from printing inks and pigments when the cardboard is made from recycled fibres recovered from printed materials. 
Table 1. Mean concentrations $\left(\mathrm{mg} \mathrm{dm}^{-2}\right)$ of heavy metals in pastry boxes packaging materials

\begin{tabular}{lccc}
\hline Heavy metals & Mean \pm SD & Range & Maximum permitted quantity \\
\hline $\mathrm{As}$ & $0.0009 \pm 0.001$ & ND-0.0019 & - \\
$\mathrm{Cd}$ & $0.00115 \pm 0.00117$ & $0.00041-0.00342$ & 0.002 \\
$\mathrm{Cr}$ & $0.018 \pm 0.019$ & $0.0088-0.0413$ & - \\
$\mathrm{Cu}$ & $0.316 \pm 0.318$ & $0.076-0.637$ & - \\
$\mathrm{Fe}$ & $4.601 \pm 4.672$ & $2.394-7.243$ & - \\
$\mathrm{Hg}$ & $\mathrm{ND}$ & $\mathrm{ND}$ & 0.002 \\
$\mathrm{Mn}$ & $0.287 \pm 0.291$ & $0.143-0.483$ & - \\
$\mathrm{Ni}$ & $0.0063-0.062$ & - \\
$\mathrm{Pb}$ & $0.016 \pm 0.017$ & $0.007-0.077$ & 0.003 \\
$\mathrm{Zn}$ & $0.037 \pm 0.038$ & $0.157-0.837$ & - \\
\hline
\end{tabular}

ND: Not detected; -: Not established

In addition, the effects of storage time and kind of pastry on the migration of heavy metals into the pastry were investigated. This was a 15-day test with interim measurements being taken at days 1,10 , and 15 at room temperature. All the migration measurements were made in duplicate. Table 2 illustrates the levels of heavy metals in pastries after different storage periods. As and $\mathrm{Hg}$ were detected in none of the samples. In all kind of pastries at time zero, $\mathrm{Fe}, \mathrm{Mn}, \mathrm{Cu}$, and $\mathrm{Zn}$ were detected at high concentrations at averages of 8.91, 4.46, 1.79 , and $1.40 \mathrm{~m} \mathrm{~kg}^{-1}$, respectively, but the differences among the means of these metals in all kind of pastries during different storage periods were not significant. On the whole, the migration of heavy metals from the recycled package to the pastry was not observed in this result except for $\mathrm{Pb}$. However, a part of the samples appeared to be contaminated with $\mathrm{Pb}$ due to their packages, as described in Table 2. These results illustrate that some heavy metals could migrate from the package when the pastry surfaces are sticky, allowing the surface of the pastry to adhere to the inner cover of the package. Further, this would allow different contaminant in the outer package layer to migrate into the pastry, because the packages were so poorly designed that their inner coatings did not maintain their structural integrity. These contaminations were higher in more sticky pastry (marmalade pastry). Storage also affected the concentration of $\mathrm{Pb}$ of pastries from days 0 to 15 , and the highest $\mathrm{Pb}$ concentration was observed on day 15 . 
MOHAMMADPOUR et al.: HEAVY METALS IN PASTRY PACKAGES

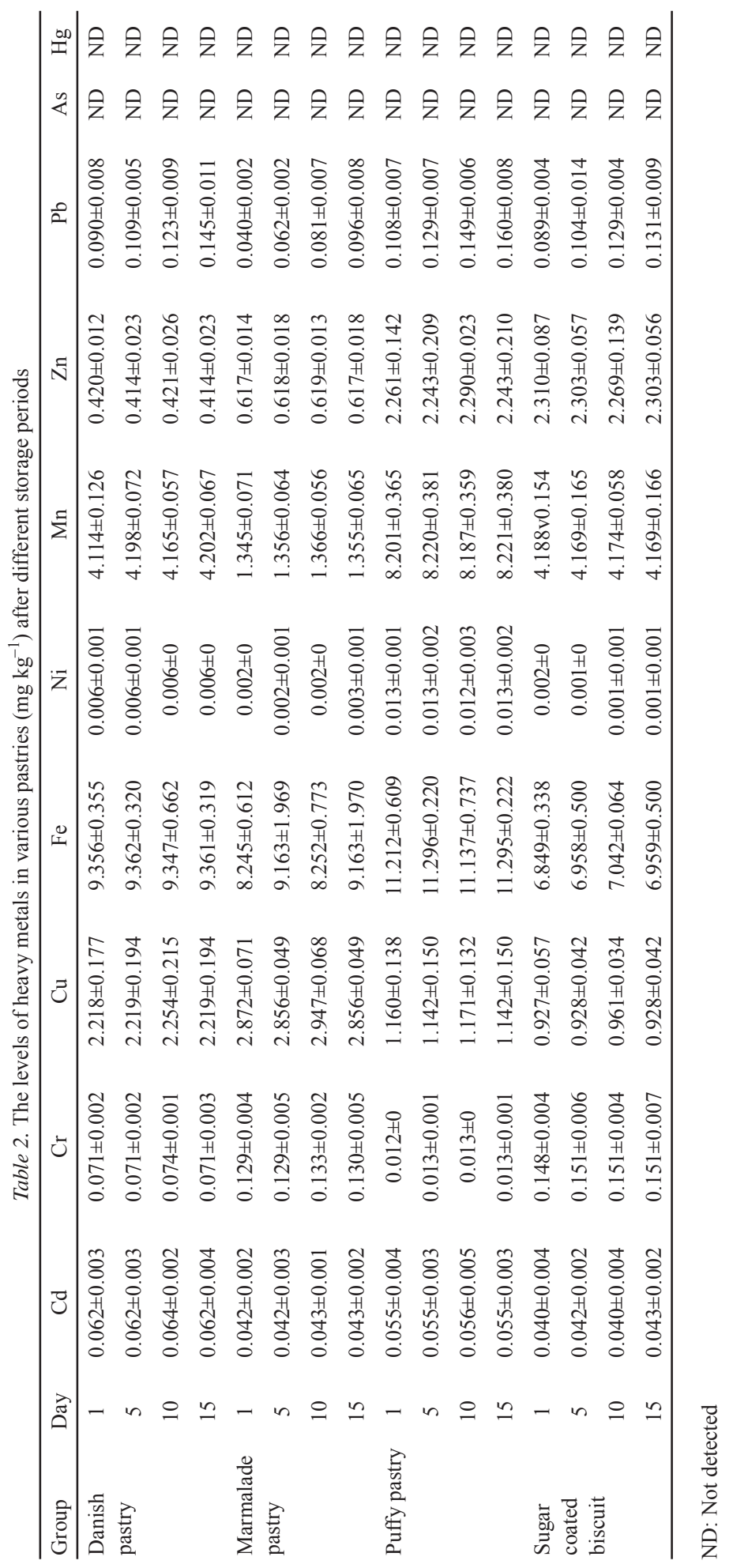




\section{Conclusions}

In this study, harmful metals such as $\mathrm{Pb}$ were detected at high concentrations in most of recycled boxes are used for pastry packaging. In addition, these results indicate that heavy metals could migrate from the recycled packages into food under real conditions of usage. Therefore, it seems that the procedure of preparing recycled paperboard for food packaging should be reconsidered. The following options are available (individually or in combination) to keep migration within acceptable limits: source control by sorting of used fibre into different grades, using of effective and improved cleaning methods such as flotation deinking, frequent testing of batches/lots, using a multi-layer barrier to prevent or slow migration, and usinhg recycled paperboard only in indirect contact applications and/or at low temperature.

This research has been supported by Tehran University of Medical Sciences grant (project No. 92-03-46-23813). Hereby, the cooperation of the University and also the Institute for Environmental Research (IER) is highly appreciated.

\section{References}

Binderup, M.-L., Pedersen, G.A., Vinggaard, A., Rasmussen, E., Rosenquist, H. \& Cederberg, T. (2002): Toxicity testing and chemical analyses of recycled fibre-based paper for food contact. Food Addit. Contam., 19, 13-28.

Снalbot, M.-C., Vei, I., Lykoudis, S. \& Kavouras, I.G. (2006): Particulate polycyclic aromatic hydrocarbons and n-alkanes in recycled paper processing operations. J. Hazard. Mater., 137, 742-751.

EC (2004): The European Parliament and of the Council on materials and articles intended to come into contact with food and repealing Directives 80/590/EEC and 89/109/EEC. OJ L 338, 13.11.2004, 4-17.

EC (2006): Good manufacturing practice for materials and articles intended to come into contact with food. Commission Regulation (EC) No 2023/2006 of 22 December 2006 OJ L 384, 29.12.2006, 75-78.

INSO (2015): Packaging - Fiber board boxes for food - Chemical specifications and test methods.1st revision. Iranian National Standardization Organization 10687, ICS:55.160. In Persian.

JAGGI, S. \& GuPTA, U. (2013): Solid phase extraction and preconcentration of Ni (II) using 1-(2-pyridylazo)-2naphthol (PAN) modified $\beta$-cyclodextrin butanediol diglycidyl ether polymer as a solid phase extractant. Maced. J. Chem. Chem. En., 32, 57-67.

JAMnicki, S., Lozo, B., RutAR, V. \& BARUŠIĆ, L. (2012): A study on the food contact suitability of recycled paper and board. Research Development, Technology. Papíripar, LVI(4), 14-20.

Khan, R., Srivastava, R., Abdin, M. \& Manzoor, N. (2013): Effect of soil contamination with heavy metals on soybean seed oil quality. Eur. Food Res. Technol., 236, 707-714.

Kim, K.-C., Park, Y.-B., Lee, M.-J., Kim, J.-B., Huh, J.-W., Kim, D.-H., Lee, J.-B. \& KiM, J.-C. (2008): Levels of heavy metals in candy packages and candies likely to be consumed by small children. Food Res. Int., 41, 411-418.

Manalili, N.M., Dorado, M.A. \& van OtterdiJK, R. (2011): Appropriate food packaging solutions for developing countries. International Congress "Save Food!” at Interpack2011, Düsseldorf, Germany. FAO, Rome. 27 pages

Nerín, C. \& Asensio, E. (2007): Migration of organic compounds from a multilayer plastic-paper material intended for food packaging. Anal. Bioanal. Chem., 389, 589-596.

Suciu, N.A., Tiberto, F., Vasileiadis, S., Lamastra, L. \& Trevisan, M. (2013): Recycled paper-paperboard for food contact materials: Contaminants suspected and migration into foods and food simulant. Food Chem., 141, 4146-4151.

Tangahu, B.V., Sheikh Abdullah, S.R., Basri, H., Idris, M., Anuar, N. \& Mukhlisin, M. (2011): A review on heavy metals (As, $\mathrm{Pb}$, and $\mathrm{Hg}$ ) uptake by plants through phytoremediation. Int. J. Chem. Eng., 2011, Article ID 939161, 31 pages. 